\title{
Recent Updates on Mesenchymal Stem Cell Based Therapy for Acute Renal Failure
}

\author{
Asmaa Missoum \\ Department of Biological and Environmental Sciences, Qatar University, Doha, Qatar
}

\author{
Key Words \\ Acute renal failure $\cdot$ Glomerular filtration rate $\cdot$ Cisplatin $•$ \\ Mesenchymal stem cells • Ischemia/reperfusion injury • \\ Acute kidney injury
}

\begin{abstract}
Acute kidney injury, formerly known as acute renal failure, is a pathological condition in which ischemia or toxic damage contributes to the loss of renal proximal tubule epithelial cells. Pathophysiological events such as oxidative stress, mitochondrial dysfunction, and direct renal tubular epithelial cells toxicity are responsible for the progression of the disease. This devastating decline in renal function affects mostly patients in the intensive care units and requires costly and invasive treatments such as dialysis and organ transplant. Fortunately, recent therapies such as the use of mesenchymal stem cells (MSCs) were proven to be effective in ameliorating renal failure via paracrine and immunomodulatory mechanisms. These fibroblast-like adult stem cells that differentiate multilineagely can be isolated from dental pulps, umbilical cords, amniotic fluids, adipose tissues, and bone marrows. Depending on their sources, the therapeutical application of each MSC type has its own capacities, advantages, and drawbacks. The review discusses and compares the latest research studies on the use of different MSCs sources to treat renal failure. Concerns about the future clinical application of MSCs such as homing, toxicity, and the risk of immune rejection are also highlighted.
\end{abstract}

\section{(c) 2020 The Author(s)}

Published by S. Karger AG, Basel

\section{Introduction}

Acute renal failure (ARF) is a type of kidney disorder where epithelial cells of the renal proximal tubule in the nephron undergo necrosis as a result of ischemia or toxic damage. This leads to sudden decrease in glomerular filtration rate (GFR), which is caused by loss of autoregulation, tubular obstruction, and increased renal vasoconstriction [1]. It affects hospitalized children at a rate of 3.9 per 1,000 admissions who are either suffering diarrhea or post-surgery renal hypoperfusion. Other conditions may include glomerulonephritis and uremic hemolytic syndrome. In case of the elderly, there is an age-dependent relationship between older age and the development of acute kidney injury (AKI). Furthermore, the mortality rate increases as the RIFLE criteria advance such that the 'risk' class has $18.9 \%$ mortality rate, 'injury' class has $36.1 \%$, and 'failure' class has $46.5 \%$ [2]. It is also believed that more than $50 \%$ patients in the intensive care units develop stage $1 \mathrm{AKI}$ and renal replacement therapy requirement is approximately $10 \%$. Unlike other kidney disorders, ARF is characterized by a sudden loss of the function, meaning that it occurs within few days or even few hours. Some of the major causes include: hypotension, heart failure, and infections. Other risk factors consist of aging, liver failure, and chronic kidney disease in addition to sepsis, shock, and exposures to nephrotoxic agents such as cisplatin [3]. Because this disorder is due to unfortunate fate and not as a result of one's health neglect, it is important to consider and

\section{KARGER}

Fax +4161306 1234

E-Mail karger@karger.com

www.karger.com
(C) 2020 The Author(s)

Published by S. Karger AG, Basel Open access

This article is licensed under the Creative Commons AttributionNonCommercial-NoDerivatives 4.0 International License (CC BYNC-ND) (http://www.karger.com/Services/OpenAccessLicense). NC-ND) (http://www.karger.com/Services/OpenAccessLicense).
Usage and distribution for commercial purposes as well as any distribution of modified material requires written permission.
Asmaa Missoum

Department of Biological and Environmental Sciences

Qatar University

Al Jamiaa St

Doha (Qatar)

E-Mail amissoum93@gmail.com 


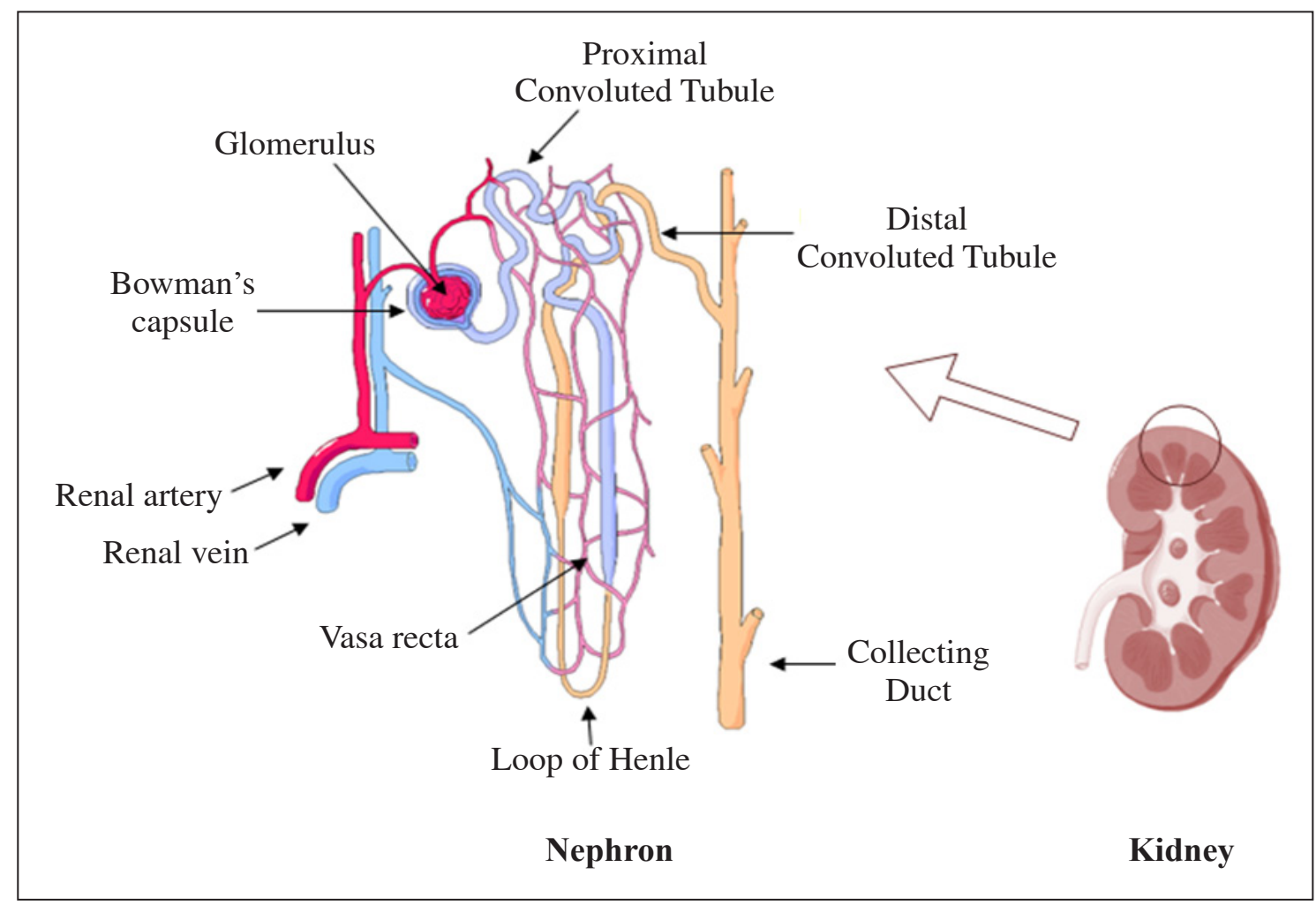

Fig. 1. Location of the nephron in the kidney and its main constitutes. The proximal tubule connects the Bowman's capsule to the loop of Henle (this figure is original for this article).

develop therapies that help patients to recover from it. Currently, treatments such as dialysis machines and organ transplantations are available, but these are costly, require continuous maintenance, and transplant organs are in high demand [4]. Therefore, other therapies such as stems cells are currently under research and clinical trials in order to provide new hope to ARF sufferers. Stem cells are special type of cells that have the capability to divide and generate differentiated cell types, which will eventually be part of either an embryonic or adult tissue [1]. Mesenchymal stem cells (MSCs), also known as mesenchymal stromal cells, are adult stem cells that are undifferentiated and can be mostly isolated from adipose tissue, umbilical cord or placenta, and bone marrow, which are able to differentiate into adipocytes, chondroblasts and osteoblasts. In addition, MSCs renoprotective effects can be due to various mechanisms such as release of paracrine signals, stimulation of local endogenous repair, and fusion or engraftment into host tissue [1]. This review discusses the recent findings regarding the ther- apeutic potentials of different types of MSCs. The main features and mechanisms of MSCs, as well as concerns about their clinical application are also mentioned.

\section{ARF Pathophysiology}

The nephron inside the kidney is composed of different types of structures in which each one of them performs an essential function. Proximal tubules (fig. 1) play a vital role in reabsorbing $65 \%$ of filtered substances such as glucose, amino acids, proteins and solutes, as well as reabsorbing $80 \%$ of filtered bicarbonates thus regulating acid-base balance thanks to important protein channels. Since ARF is mainly characterized by ischemic damage of renal proximal tubules, the epithelial tubule cells and the brush border membranes are shedded and excreted into the urine. As a result, the function of proximal tubule is lost as well [2]. Proximal tubular injury involves apoptosis, cell-cycle proteins dysregulation, DNA damage, autophagy, activation of mitogen-activated protein kinase signaling pathways, and direct renal tubular epithelial 
cells toxicity. Oxidative stress is an important pathophysiology mechanism in which reactive oxygen species are generated, lipid peroxidation products are accumulated in kidneys, and antioxidant systems are suppressed [5]. In addition, the inflammation mechanism contributes to secretion of cytokines, interleukin-33 (IL-33) and IL-10, an increase in both serum and urine TNF- $\alpha$ concentrations, and the infiltration of $\mathrm{T}$ cells, macrophages, mast cells, neutrophils to the kidney tissue. Lastly, vascular injury involves impaired vascular autoregulation and endothelial dysfunction that result in renal vasoconstriction [5]. Mitochondria dysregulation is another early event that precedes cell death. In fact, a high mitochondrial density resides in all nephron segments and proximal tubular epithelium due to their crucial role in active transport, which necessitate high-energy. What's more, microtubules have been shown to regulate the intracellular distribution of mitochondria [6]. When membrane depolarization and permeabilization is combined with mitochondrial fission, apoptogenic factors related to the generation of radical oxygen species are released, making tubular cells the most important targets of AKI. This leads to ATP depletion and cytoskeletal alterations causing cell-to-cell contact loss, brush border breakdown, and detachment of tubular epithelial cells. Thus, the phenomenon of mitochondria dysregulation is responsible for the progression of AKI [6].

\section{MSC Sources, Characteristics, and Mechanisms}

MSCs are classified into several types according to their sources. They can be isolated from gingiva, tooth pulps, periodontal tissue, umbilical cord, amniotic fluid, Wharton's jelly, adipose tissue, bone marrow, and even skeletal muscle tissue and liver tissue. Yet, MSCs from adipose tissue are the most widely researched after bone marrow MSCs (MB-MSC), because of their abundance and their easy extraction compared to MB-MSCs [7]. Generally, MSCs are fibroblast-like cells that are adherent and capable of multilineage differentiation as well as self-renewal. Their isolation methods, which include selection (cell sorting and adherence to plastic), expansion (oxygen tension and culture media), and other enzymatic or nonenzymatic tests, are not fully standardized until now. In 2006, the International Society for Cellular Therapy recommended certain criteria to uniformize MSCs characteristics, isolation, and cultivation in clinical applications [8]. The criteria describes MSCs features as: ability to adhere to plastic surfaces and to differentiate into

Mesenchymal Stem Cell Based Therapy for Acute Renal Failure osteocytes, chondrocytes, and adipocytes under standard in vitro conditions. These include lack of the hematopoietic markers such as CD11b, CD14, CD34, CD45, CD79a and HLA-DR, in addition to surface expression of CD73, CD90, and CD105 [9].

Furthermore, MSCs exhibit immunoregulatory properties such as prompting T-cell expansion to a regulatory phenotype, shifting macrophages to anti-inflammatory and immunosuppressive M2 phenotypes, and inhibiting antigen-presenting cells [9] and dendritic cells maturation. The functions of NK cells, B cells, and memory T cells are suppressed as well. This is an advantage as differentiated renal cells from this therapy are less likely to be rejected by the immune system of the patient, unlike when performing organ transplant, which requires immunosuppressive drugs [10]. The MSCs migrate to the damaged renal tissue and secrete a number of cytokines and chemokines that can correct the course of injury. They regulate the immune response caused by tissue repair through paracrine in addition to endocrine mechanisms [11]. Moreover, some studies suggest that they are capable of fusing with resident tubular cells and several others suggest that they are capable of differentiating into resident kidney cells [12].

Hypothetically after transplantation, MSCs enter the blood circulation expressing a variety of adhesion molecules such as L-selectin, P-selectin, and vascular cell adhesion molecule-1. Consequently, the activated neutrophils adhere to the vascular endothelium and enter the renal interstitium, resulting in reduced neutrophil infiltration [13]. MSCs also reduce inflammation, induce prosurvival genes, and down-regulate pro-apoptotic genes. In order to activate endogenous cell repair, MSCs release the following factors: vascular endothelial growth factor, stromal cell-derived factor-1, fibroblast growth factor, insulin-like growth factor (IGF), keratinocyte growth factor, hepatocyte growth factor, and monocyte chemoattractant protein-1. Their microvesicles, however, transfer mitochondria into the damaged cells to restore adenosine triphosphate supply after injury [9].

Indeed, MSCs exosomes and derived microvesicles have been pointed out as a recent mechanism of cell-tocell communication, which allows the transfer of genetic material or functional proteins via mRNAs and microRNAs. For example, IGF-1, which has anti-apoptotic properties, has been investigated in terms of its contribution to direct proliferation of damaged proximal tubule cells through cell-to-cell communications [14]. Researchers derived exosomes and macro-vesicles from MSCs and enhanced the delivery of IGF-1 receptor mRNA through 
cell activation and local IGF-1 production. MSCs were also modified genetically by retroviral vectors that encode for CXCR4, a homing receptor, to improve their migratory behavior. This has resulted in proliferation of renal proximal tubule cells and improved regenerative effects. In contrast, the knocking down of IGF-1 expression by siRNA before infusion did not result in cell protective effects of MSCs [14]. Other different growth factors produced by paracrine mechanisms include monocyte-chemoattractant protein-1, vascular endothelial growth factor, basic fibroblast growth factor, and hepatocyte growth factor [15].

\section{Using MSCs to Treat ARF}

\section{MSCs Derived from Dental Pulp}

Stem cells from human exfoliated deciduous teeth (SHED), which are considered as a medical waste, can be easily isolated from dental pulps. They share similar properties with BM-MSCs such as the ability to proliferate and differentiate multipotentially. However, unlike BM-MSCs, SHED possesses advanced biomaterials interactivity that utilized in tissue engineering applications, in addition to a high proliferative capacity. In a recent study, SHED have managed to lower serum creatinine, cytokine levels (MIP-2, MCP-1, and IL-1 $\beta$ ), and blood urea nitrogen levels compared to BM-MSCs, which showed no therapeutic effect in mice model of AKI. In vitro SHED significantly reduced $\mathrm{MCP}-1$ secretion in tubular epithelial cells and promoted wound healing [11]. Dental pulp stem cells were also proven in vitro to maintain their stemness under extracellular acidosis conditions. It was found that the downregulation of $R U N X 2$, which is an osteoblast-related gene, combined with a slight augmentation of $S O X 9$ expression, advocates a less differentiated osteogenic phenotype [16].

\section{MSCs Derived from Embryos}

Fetal MSCs have low immunogenicity and do not contribute to hyperplasia, which is common in adult stem cells treatment. Researchers showed that human embryonic MSCs injected into newborn mice's kidneys may transform to renal tubule epithelium. After 2 weeks, the percentage of PKH26-labeled MSCs localized to the renal tubules was $10 \pm 2.1 \%$ as determined by laser scanning confocal microscopy. Although PKH26 labeling was used, it has no effect on mesenchymal cell surface features, human MSC differentiation, and proliferation. However, the use of MSCs from human embryos could raise eth- ical and safety issues, especially when obtained from voluntary terminations of pregnancy. For this reason, research on embryonic MSCs remains limited even though fetal MSCs have a high proliferative capability [17].

\section{MSCs Derived from Amniotic Fluid}

Human amniotic fluid derived MSCs (hAF-MSCs) have a broader differentiation capacity and higher proliferation rates when compared to adult stem cells such as adipose or bone marrow derived stem cells [12]. Compared to embryonic stem cells, the use of hAF-MSCs is considered ethically acceptable as it is easily available without destruction of human embryo. Because they are genomically stable, hAF-MSCs lack teratoma formation when injected in vivo [18].

Treatment with hAF-MSCs have managed to reduce serum creatinine levels after $24 \mathrm{~h}$, as well as to cause higher proliferation index, less inflammatory cell infiltration, less myofibroblasts and less tubular necrosis at $48 \mathrm{~h}$. The cells were delivered intra-arterially in rats 6 $\mathrm{h}$ after ischemia/reperfusion (I/R) injury. It is concluded that hAF-MSCs contain a renal progenitor cell subpopulation that has a nephroprotective effect [12].

In Sprague-Dawley rats with cisplatin-induced nephrotoxicity, hAF-MSCs significantly reduced oxidative stress, improved renal function, and increased the kidneys' regenerative capacity. These protective and regenerative activities could be mainly due to an antioxidant activity [18].

\section{MSCs Derived from Umbilical Cord}

MSCs derived from human umbilical cord (UC-M$\mathrm{SCs}$ ) can be collected easily providing a new extensive source of MSCs. Therefore, this transforms medical waste into beneficial products for clinical applications [13]. Compared to other MSCs, UC-MSCs have low immunogenicity that prevents immune rejection in allogeneic transplantation. Besides, they exhibit a high potential of multilineage differentiation and higher rate of colony-forming unit fibroblasts [19].

In rats with renal I/R injury, treatment with UC-MSCs by caudal vein injection showed less histopathological damage to the renal tissues compared to other groups. Moreover, the expression of ICAM-1 and the number of polymorphonuclear neutrophils were reduced significantly after $48 \mathrm{~h}$. It was hypothesized that UC-MSCs partially inactivated ICAM-1 and decreased neutrophils infiltration. Hence, endogenous repair was initiated by an inflammatory cascade [13].

In male rats with I/R injury, UC-MSCs improved GFR 
and tubular function better than adipose derived MSCs. The treatment also contributed to higher expression of aquaporin-2 and Klotho, as well as to down regulation of microRNAs; microR-29a and microR-34a, and senescence-related proteins such as $\beta$-galactosidase, p21Waf1/ Cip1, and p16INK4a. This was investigated as some microRNAs have been associated with cellular senescence, since they promote redox imbalance. Nevertheless, UC-MSCs have been shown to be more effective in reducing the expression of cell-cycle inhibitors than those derived from adipose tissue [20].

Interestingly, applying a combination of UC-MSCs with $20 \mu \mathrm{mol} / \mathrm{l}$ of resveratrol to cisplatin-induced AKI model demonstrated a more efficient repairing effect than did UC-MSCs. Resveratrol-promoted UC-MSCs resulted in the downstream phosphorylation of extracellular signal-regulated kinase due to secreted platelet-derived growth factor-DD into renal tubular cells. This inhibited renal tubular cells apoptosis. In vitro the conditioned medium accelerated vascular endothelial cells proliferation and migration, thus promoting angiogenesis [21].

Using the same AKI model, UC-MSCs enhanced PGC1 $\alpha$ expression, Sirtuin 3 (SIRT3) activity, and NAD+ biosynthesis resulting in mitochondrial biogenesis regulation in proximal tubuli. In SIRT3-deficient mice with AKI, the UC-MSC treatment did not stimulate renoprotection. This means that SIRT3 might play an important role in tubular recovery, possibly through mitochondrial dynamics preservation [6].

According to biochemical parameters and histological analysis, the use of UC-MSCs to treat gentamicin-induced AKI in rats was shown to be more effective through the over-expression of IGF-1. This was clarified by RNA microarray analysis, which confirmed the upregulation of key genes associated with cell migratory, anti-oxidation, and anti-inflammatory capacities. This modification of UC-MSCs was established through retroviral infection [19].

\section{MSCs Derived from iPS Cells}

Induced pluripotent stem (iPS) cells are mature, adult cells that are reprogrammed to express certain transcription factors that play a chief role in determining their state. These cells have similar differentiation and self-renewal properties to those of embryonic stem cells. MSCs derived from iPS cells (iPS-MSCs) are limitless because iPS cells display unlimited proliferation capacity and can be induced from any somatic cells in the body. Traditional MSCs, on the other hand, have limited accessibility because of the paucity of their population in the tis-

Mesenchymal Stem Cell Based Therapy

for Acute Renal Failure sue and the tedious procurement procedure [22]. In mice with adriamycin-induced nephropathy, injections of iPS-MSCs significantly reduced intra-renal deposition of $\alpha$-smooth muscle actin and collagens I and IV, attenuated oxidative stress, and inhibited tubulointerstitial fibrosis. The anti-fibrotic effect of iPS-MSCs was due to hedgehog signaling pathway modulation. In vitro conditioned medium from iPS-MSCs significantly reduced expression of tubular Bax and tubular apoptosis, raised expression of Bcl2 and survivin, along with an enhancement in tubular proliferation [22].

Similarly, iPS-MSCs provided renoprotective effects in male-adult Sprague-Dawley rats with I/R injury, as the architectures of podocyte components, glomeruli, and renal tubules were preserved. It was found that protein expressions of angiogenesis markers such as VEGF, CXCR4, and SDF-1 $\alpha$ were increased in kidney parenchyma [23].

Adult peripheral blood mononuclear cells were reprogrammed into induced MSCs using an oocyte suspension. In rabbits with unilateral ureteral obstruction, both kidney tissue fibrosis and pathological parameters of blood biochemistry were reduced, in addition to increased GFR and restored renal blood flow, after 4 weeks of cell transplantation therapy. In this method, there was high induction efficiency and no risk for gene transfer, culture toxicity or damage to the cells, as seen in gene transfection with synthetic chemicals [24].

\section{MSCs Derived from Adipose Tissue}

MSCs derived from adipose tissue (AD-MSCs) are highly abundant in adipose tissue and can be easily extracted as liposuction is widely used clinically. Their allogeneic transplantation via the intra-renal route contributed to a low degree of necrosis and higher vascularization of the renal parenchyma in Wister rats [26]. Besides, the proliferation rate of AD-MSCs is higher by 40-fold than that of BM-MSCs [15].

In Sprague-Dawley rats with cisplatin-induced AKI, AD-MSCs were proven to have the least effective in regenerative and proliferative activities, compared to BM-MSCs and AF-MSCs [26].

However, using the same study model, AD-MSCs have improved tubular cells necrosis and renal failure via the secretion of specific cytokines. These were able to hinder p38 MAPK activation, which in turn modulated the expression levels of Bcl-2 and Bax to the normal level. Subsequently, the apoptosis pathway was inhibited. It is hypothesized that injured tubular cells release certain signals, which trigger AD-MSCs to produce these spe-

Curr Urol 2019;13:189-199 
cific cytokines [28]. In addition, AD-MSCs have limited renal fibrosis development largely through an anti-oxidative activity. They have attenuated the decrease in glutathione, superoxide dismutase, and creatinine clearance caused by cisplatin [28].

The administration of AD-MSCs via the tail vein have resulted in a significant decrease in serum and tissue malondialdehyde levels, in Sprague-Dawley rats submitted to renal I/R injury. This may act through an antioxidative activity followed by an anti-inflammatory effect [29]. Using the same transplantation method, rat AD-MSCs have also managed to reverse cisplatin-induced nephrotoxicity effects in adult female Wister rats. The tubular cell proliferation was increased and both interstitial inflammation and fibrosis were reduced. The MSCs also differentiated into tubular epithelial cells [15].

It is also important to mention that modifying AD-MSCs or their microenvironment could enhance the efficacy of their therapy. This was demonstrated in several recent studies. For example, hypoxia preconditioning ameliorated AD-MSCs paracrine effects such as angiogenic factors, as well as antioxidative activities, serum creatinine and blood urea nitrogen levels. This was carried in vitro and in vivo (rats with ischemia-reperfusion). Thus, hypoxia may have activated MSCs anti-apoptotic capacity, which resulted in further anti-apoptotic cytokine release [30].

Moreover, aggregating AD-MSCs into 3D spheroids enhances their survival rate, secretion of angiogenic factors, and the expression of surface antigens, which facilitate their homing in a new environment. When compared to $2 \mathrm{D}$ cultured cells, AD-MSCs 3D spheroids reduced tissue damage to a greater extent, which promoted vascularization and improved renal function in I/R injury rats. In vitro studies also confirm that MSCs 3D spheroids exhibited stronger anti-apoptotic and anti-oxidative capacities, and produced more extracellular matrix proteins [31].

Another strategy to boost therapeutic potential of AD-MSCs is to pretreat it with other biochemical compounds. In vitro pretreatment of human AD-MSCs with melatonin $(100 \mu \mathrm{M}$ for $3 \mathrm{~h})$ has significantly amplified their proliferation and their expression of antioxidative enzymes (catalase and heme oxygenase 1), and pro-survival (P-Akt and P-Erk1/2). Consequently, this improved migration and proliferation of HK-2 human kidney epithelial cells that were exposed to cisplatin [32].

Exendin-4 is a glucagon-like peptide that has an essential hypoglycemic function. When it was therapeutically combined with AD-MSCs, kidney $\mathrm{I} / \mathrm{R}$ in male
Sprague-Dawley rats was significantly improved. The circulating levels of blood urea nitrogen and creatinine were lower than those of AD-MSCs-only group (p $<0.0001$ ). It is also important to mention that this AKI model was complicated by severe sepsis syndrome. The exact mechanism that contributed to this study's results is not entirely clear and therefore further investigation is required [33].

Although MSCs migrate to the injured tissues, they are only detectable at very low frequencies. Therefore, the fate of AD-MSCs was investigated using bioluminescence imaging and qRT-PCR. In Albino C57BL/6 mice with cisplatin induced AKI, it was found that qRT-PCR was more convenient for tracking cells after transplantation [34].

\section{MSCs Derived from Bone Marrow}

BM-MSCs constitute $0.01 \%$ of total cells and regulate self-renewal and maturation. They also recruit hematopoietic stem cells to the vascular compartment. This is achieved through secretion of chemokines, cytokines, and growth factors or cell-to-cell interaction. They also regulate self-renewal and maturation [35].

Using Sprague-Dawley rats as study models, it was shown that the route of BM-MSCs administration does not have a significant influence on therapy as intra-arterial, intravenous, and kidney sub capsular injections resulted equally in kidney function and tissue damage amelioration [36]. The BM-MSCs treatment in cisplatin and I/R injury induced AKI has resulted in lower lipid peroxidation, a similar effect of diphenyl-p-phenylenediamine antioxidant therapy [37].

Under hypoxic condition, the treatment had led to an optimal recovery of renal biochemical parameters, which suggests that it plays a role in the preservation and the survival of MSCs [38]. The treatment is also effective when it is applied prior to the expansion of an inflammatory microenvironment. Urine neutrophil gelatinase-associated lipocalin may be useful for detecting inflammation [39].

BM-MSCs were more effective in AKI treatment induced by cisplatin in Wister rats compared to candesartan, which an angiotensin II blocker. BM-MSCs significantly reduced serum creatinine and urea levels, apoptotic markers such Bax and caspase-3, as well as inflammatory markers such as p38MAPK, TNF- $\alpha$ and MCP-1 [40]. In case of I/R injury induced AKI, there were enhancements in histological and biochemical parameters via both intra-parenchymal and intra-aortic routes [41].

Moreover, the overexpression of a nuclear erythroid 
2-related factor 2 (Nrf2), which is a cytoprotective factor that induces antioxidant enzymes transcription, had improved renal histology, serum creatinine, and urea levels more than unengineered BM-MSCs [42]. Highly similar outcomes were confirmed in a glycerol-induced AKI study where the overexpression of Nrf2 in MSCs protected them against apoptosis and boosted their viability. Therefore, this could suggest a new strategy to enhance the effectiveness of BM-MSCs therapy [43]. However, the packing of BM-MSCs with Duragen has reduced apoptosis, ameliorated tubular lesions, and preserved renal function in C57BL/6 mice with glycerol-induced AKI complicated by rhabdomyolysis. This was due to the activation of PI3K/Akt pathway and inhibiting cell apoptosis [44].

The homing of BM-MSCs was also investigated by transforming growth factor- $\beta 1$ (TGF- $\beta 1$ ). The overexpression of TGF- $\beta 1$ has regulated CXCR 4 expression, the only known receptor of SDF-1, on cell membranes in Sprague-Dawley rats with I/R injury. Thus the BM-MSCs migration by SDF-1/CXCR4 axis was enhanced, which resulted in ameliorated renal tissue damage [45]. Similarly, the overexpression of heme oxygenase-1 lowered acute tubular necrosis and enhanced BM-MSCs proliferation and differentiation possibly by increasing PCNA+ and CK18+ levels. These effects could be due to the stimulation of PI3K/Akt and MEK/ERK signaling pathways [46].

Direct injection of MSCs into target arteries or organs is very difficult, intravenous administration is widely used for cellular therapies. Although several strategies were developed to control cell homing both spatially and temporally, they are imprecise and invasive. In $\mathrm{C} 3 \mathrm{H}$ mice with cisplatin-induced AKI, the homing of BM-MSCs was improved via pulsed focused ultrasound. This noninvasive image-guided strategy resulted in enhanced regeneration and renal function as well as reduced tubular cell death [47].

Interestingly, a short clinical study was conducted on BM-MSCs therapy such that 11 chronic renal failure patients were injected with their respective MSCs, which were extracted and harvested from their bone marrow samples. This was carried out by transfemoral catheter every week for a period of 6 months. After followed-up there was a significant progression in GFR, blood urea, and creatinine levels $(p=0.000)$. Despite the small study size, BM-MSC therapy provided hope for patients who were on regular hemodialysis through tissue regeneration [10]. Nevertheless, in non-human primate study models such as monkeys (Macaca fascicularis), I/R injury-in-

Mesenchymal Stem Cell Based Therapy for Acute Renal Failure duced AKI complicated by diabetes mellitus was ameliorated using human BM-MSCs. According to histological analysis, tubular damage was recovered and qRT-PCR findings showed lower neutrophil gelatinase-associated lipocalin mRNA expression, a protein that acts as an AKI marker. Since the renal structure differs between mice and humans, it is important to use animal models apart from rodents to better interpret preclinical findings [48].

As mentioned earlier, the number of adult MB-MSCs is extremely low and depends on donors. Usually ranging from 0.01 and $0.00001 \%$ of total mononuclear cells, their quantity and differentiation also decline with increasing age [17].

\section{Extracellular Vesicles from MSCs}

In order to initiate paracrine action on kidney regeneration, MSCs release extracellular vesicles (EVs). These are minute extracellular membrane fragments, which are heterogeneous for their content, origin, and dimensions. The EVs sub-populations can be separated by gradient floatation and differ in proteomic and miRNomic profiles. Hence, some exosomal markers are more active than others on protection from apoptosis, as well as on proliferation of renal tubular cells [49]. EVs are classified into 2 distinct types: exosomes, ranging $40-100 \mathrm{~nm}$ in diameter, are formed inside the endosomal network and larger microvesicles having a diameter of 100-1,000 $\mathrm{nm}$, are generated by the outward budding of plasma membrane [50].

The biodistribution of EVs and their renal localization in CD1 mice with glycerol-induced AKI were investigated using optical imaging. It was found that directly labeled EVs exhibited a higher fluorescence and generated more preserved signal than labeled EVs produced by pre-treated cells. This was useful in tracking MSCs kinetics [51].

In SCID mice with I/R injury, EVs from glomerular MSCs activated tubular epithelial cell proliferation. According to miRNome analysis, these vesicles selectively expressed a group of microRNAs, which suggests their capacity to induce epigenetic changes in target cells. This was further demonstrated by the ineffectivity of RNase-inactivated EVs [52].

Similarly, in male Sprague-Dawley rats with I/R injury, iPS-MSCs secrete EVs, which contribute to anti-necroptosis effects by fusing with renal cells to deliver specificity protein. Consequently, the expression of sphingosine kinase 1 is transcriptionally activated to increase sphinganine-1-phosphate formation [53]. Both in vitro and in vivo, hepatocyte growth factor expression 


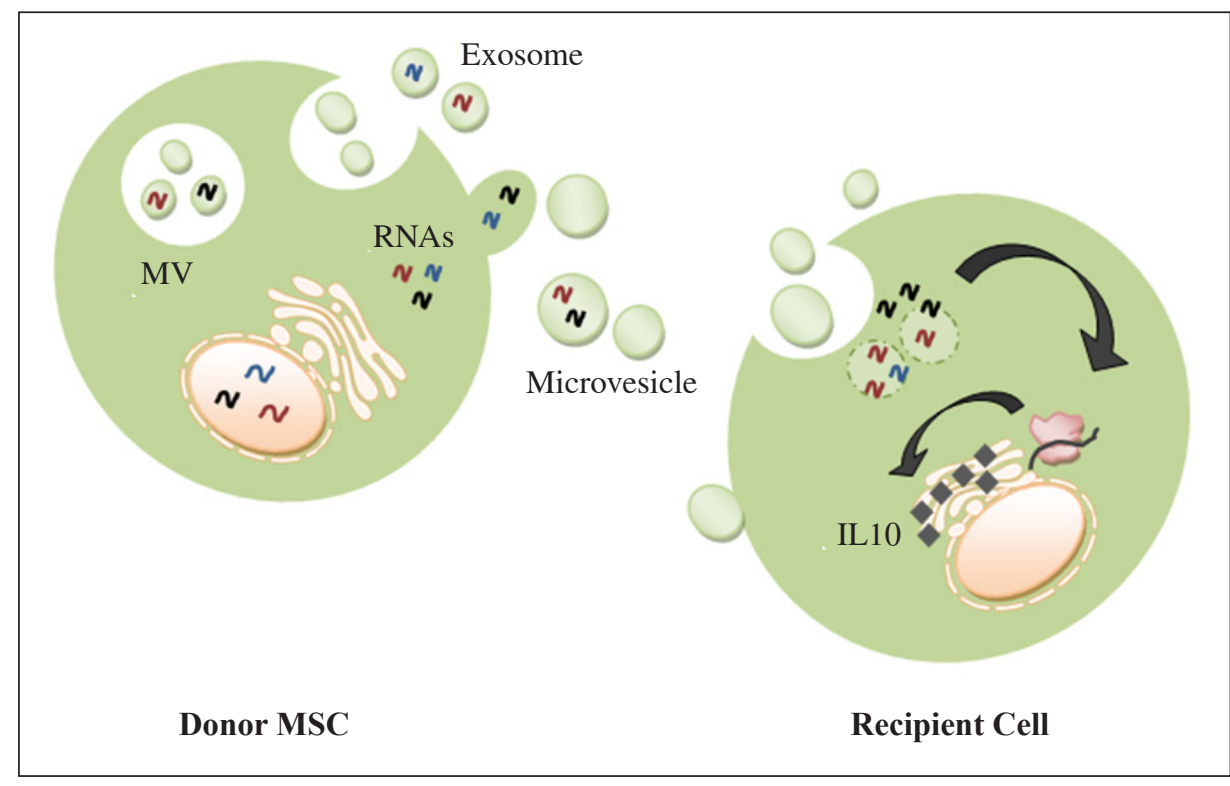

Fig. 2. Horizontal transfer of EV-embedded mRNA as part of paracrine mechanism (this figure is original for this article).

in damaged rat tubular cells was significantly enhanced by microvesicles treatment from human UC-MSCs. This strongly stimulated tubular cell dedifferentiation, which is an important regenerative mechanism [54].

Wharton's Jelly MSCs EVs also play an anti-oxidative role by enhancing Nrf2/ARE activation both in vivo (using rats) and in vitro. $\mathrm{Nrf} 2$ is a transcription factor that, when activated, enters the nucleus and binds to antioxidant response elements, and thus modulating the expression of large numbers of antioxidants such as HO-1 up-regulation [55]. Furthermore, EVs derived from human Wharton's Jelly MSCs have inhibited mitochondrial fission via miR-30b/c/d and reduced cell apoptosis, therefore ameliorating acute renal I/R injury in rats. This was investigated by tracking the expression of dynamin-related protein 1 , a vital regulator of mitochondrial fission [56].

AD-MSCs EVs has upregulated tubular Sox 9 expression in C57BL/6 mice with unilateral renal I/R injury. Therefore, tubular regeneration was promoted by activating receptors of Sox9 on tubular epithelial cells. Sox9 is a transcription factor that plays a crucial role in the development of kidneys [57].

In a recent study, EVs from UC-MSCs were proven to have faster kinetics than those of BM-MSCs. More- over, kidney tubular cells lacking IL-10 expression acquired the IL-10 mRNA after treatment from both types of MSCs (fig. 2). The mRNA was efficiently translated into the corresponding protein. At tubular cell level, this may enhance the IL-10/IL-10R1R2 anti-inflammatory pathway [50].

The effect of microRNA deregulation on regenerative potential of MSCs EVs was also evaluated. In murine models of glycerol-induced AKI, Drosha-knockdown cells produced EVs analogous to those of wild-type cells, but were ineffective in promoting AKI morphological and functional recovery, when administered intravenously. This means that microRNAs from MSCs EVs have a critical role in recovery after AKI [58].

\section{Concerns about Clinical Application}

Regarding MSCs clinical application in AKI, there are several concerns that must be taken in account. First, the conditions of cell culture may contribute to toxicity. For instance, MSCs can acquire chromosomal abnormalities with expansion. These changes must be monitored during cell manufacturing procedures, in which master cell banks are generated. In an over-passaged line, a senescent population of cells may emerge causing cells to be- 
have differently from the original population [59]. Secondly, if MSCs used are not autologous (meaning that they are host-derived), then the recipient will require a suitable immunosuppressive therapy to prevent the risk of immune rejection. However, the harvest of autologous MSCs involves complex processes, timing constraints, and high expenses. In addition, if they are obtained from older donors, there are high chances for increased apoptosis signals and lower proliferation and differentiation capacities. In effect, MSCs can be immunoprivileged in vivo provided the lack of major histocompatibility class II antigens. Many experimental findings proved that autologous MSC therapy can be safe and effective after AKI [60]. The third concern is the risk of tumorigenicity. At the present, there is no evidence of tumor formation that has been observed in animal studies or even in human trials. It is difficult to detect tumor formation in rodent models due to low human MSCs transformation rate. In vitro uremic toxins impaired the function human BM-MSCs by altering MSCs paracrine activity, but not by promoting apoptosis. This alarms on their possible role in inducing MSCs malignant trans-differentiation [61]. Finally, only $1 \%$ of the injected cells reach the target site, whereas most are trapped in the lungs, spleen and liver. MSCs either cannot find their way, or simply die due to the in vivo harsh microenvironment resulting in low number of transplanted MSCs in kidneys. For this reason, preconditioning methods have been used to improve MSCs homing, which is a major concern [60].

Some limitations concerning the clinical application of MSCs for AKI will be barely resolved in the future. For example, it is more ideal to apply cells at the exact moment of AKI onset. Unfortunately there are no specified symptoms or biomarkers that allow early and fast AKI detection. In case of patients with sepsis or heart failure, the situation is more devastating as it is almost impossible to diagnose AKI early on [62]. Moreover, it is still unknown which optimal dose and route of delivery has the most therapeutic effect in clinical trials. In general, time-sequence, optimal dose, and route of administration remain to be determined.

\section{Future Perspectives}

Current experimental evidence confirms that MSCbased therapy contribute to cellular repair in renal injury. However, the role of MSCs can be exerted clinically only if their survival, paracrine, and migration capacities are improved. Due to their heterogeneity, it is difficult to choose the best preconditioning strategy as it depends on sources and donors, doses, and route timing of transplanted MSCs. Therefore, it is important to explore the molecular and cellular mechanisms of the preconditioning strategy in order to expand its application and avoid its side effects in AKI. Moreover, concerns of long-term safety are generally based on outcomes of animal studies. Many experiments reported that $67.2 \%$ injected MSCs end up in the lungs compared to only $5.4 \%$ in the kidneys 1 day after injection [63]. In this case, MSCs paracrine mechanism of actions that mediate renal repair could be therapeutic alone. Last but not least, mitochondrial transfer from MSCs can possibly serve as an effective remedy to treat conditions with mitochondrial dysfunction such as AKI. There are very few studies that have investigated mitochondrial transfer, which suggest that it can function independent of paracrine effects [64]. As mentioned previously, MSCs microvesicles were shown to manage oxidative stress by targeting depolarized mitochondria. This suggests a cross-talk between MSC paracrine factors and mitochondria. Because the combinatorial effect of these therapies is largely unexplored, a systematic study is essential to completely assess the safety profile and efficacy of MSCs in AKI.

\section{Conclusion}

In conclusion, $\mathrm{ARF}$ is a devastating disorder that is characterized by necrosis of proximal tubule cells in the nephron that carry out important functions such as water and solutes reabsorption. Because available treatments such as hemodialysis and organ transplant are not effective in treating this condition according to previously discussed limitations. AF-MSCs therapy seems to have promising effects on ARF rodent models, as their properties qualifies them more compared with other types of MSCs. However, research on AF-MSCs is still limited due to ethical issues. AD-MSCs and BM-MSCs based therapies have been also shown to have efficient effects on experimental ARF according to recent research. Moreover, the use of recent strategies has improved their homing, survival and proliferation both in vivo and in vitro. This includes delivery of growth factors, receptors and their mRNAs through macrovesicles and retroviral vectors, bearing in mind safety issues. Regardless certain complications with MSCs, it is essential to carry out human MSCs therapy trials whilst considering ethical issues. New discoveries could change the scope of future regenerative medicine. 


\section{References}

-1 Du T, Zhu YJ: The regulation of inflammatory mediators in acute kidney injury via exogenous mesenchymal stem cells. Mediators Inflamm 2014;2014:261697.

$>2$ Makris K, Spanou L: Acute kidney injury: definition, pathophysiology and clinical phenotypes. Clin Biochem Rev 2016;37:85-98.

$\checkmark 3$ Bellomo R, Ronco C, Mehta RL, Asfar P, Boisramé-Helms J, Darmon M, Diehl JL, Duranteau J, Hoste EAJ, Olivier JB, Legrand M, Lerolle N, Malbrain MLNG, Mårtensson J, Oudemans-van Straaten HM, Parienti JJ, Payen D, Perinel S, Peters E, Pickkers P, Rondeau E, Schetz M, Vinsonneau C, Wendon J, Zhang L, Laterre PF: Acute kidney injury in the ICU: from injury to recovery: reports from the 5th Paris International Conference. Ann Intensive Care 2017;7:49.

$\checkmark 4$ Aghajani Nargesi A, Lerman LO, Eirin A: Mesenchymal stem cell-derived extracellular vesicles for kidney repair: current status and looming challenges. Stem Cell Res Ther 2017;8:273

5 Ozkok A, Edelstein CL: Pathophysiology of cisplatin-induced acute kidney injury. Biomed Res Int 2014;2014:967826.

-6 Perico L, Morigi M, Rota C, Breno M, Mele C, Noris M, Introna M, Capelli C, Longaretti L, Rottoli D, Conti S, Corna D, Remuzzi G, Benigni A: Human mesenchymal stromal cells transplanted into mice stimulates renal tubular cells and enhance mitochondrial function. Nat Commun 2017;8:983.

7 Ayala-Cuellar AP, Kang JH, Jeung EB, Choi $\mathrm{KC}$ : Roles of mesenchymal stem cells in tissue regeneration and immunomodulation. Biomol Ther (Seoul) 2019;27:25-30.

$>8$ Peired AJ, Sisti A, Romagnani P: Mesenchymal stem cell-based therapy for kidney disease: a review of clinical evidence. Stem Cells Int 2016;2016:4798639.

-9 Erpicum P, Detry O, Weekers L, Bonvoisin C, Lechanteur C, Briquet A, Beguin Y, Krzesinski JM, Jouret F: Mesenchymal stromal cell therapy in conditions of renal ischaemia/reperfusion. Nephrol Dial Transplant 2014;29:1487-1493.

10 Gabr H, Zayed RA: Mesenchymal stem cell infusion in chronic renal failure patients. J Med Bioengineering 2015;4:329-331.

$\checkmark 11$ Hattori Y, Kim H, Tsuboi N, Yamamoto A, Akiyama S, Shi Y, Katsuno T, Kosugi T, Ueda M, Matsuo S, Maruyama S: Therapeutic potential of stem cells from human exfoliated deciduous teeth in models of acute kidney injury. PLoS One 2015;10:e0140121.

-12 Monteiro Carvalho Mori da Cunha MG, Zia S, Oliveira Arcolino F, Carlon MS, Beckmann DV, Pippi NL, Luhers Graça D, Levtchenko E, Deprest J, Toelen J: Amniotic fluid derived stem cells with a renal progenitor phenotype inhibit interstitial fibrosis in renal ischemia and reperfusion injury in rats. PLoS One 2015;10:e0136145.
13 Qiu Z, Zhou D, Sun D: Effects of human umbilical cord mesenchymal stem cells on renal ischaemia-reperfusion injury in rats. Int Braz J Urol 2014;40:553-561.

14 Morigi M, Benigni A: Mesenchymal stem cells and kidney repair. Nephrol Dial Transplant 2013;28:788-793.

15 Sakr AE, Elhai WA, Zeid AA, Ali H: Transplanted adipose derived mesenchymal stem cells attenuate the acute renal injury induced by cisplatin in rats. Egypt J Histol 2017; 40:169-183.

16 Massa A, Perut F, Chano T, Woloszyk A, Mitsiadis TA, Avnet S, Baldini N: The effect of extracellular acidosis on the behaviour of mesenchymal stem cells in vitro. Eur Cell Mater 2017;33:252-267.

17 Yuan L, Liu HQ, Wu MJ: Human embryonic mesenchymal stem cells participate in differentiation of renal tubular cells in newborn mice. Exp Ther Med 2016;12:641-648.

18 Al-Husseiny F, Sobh MA, Ashour RH, Foud S, Medhat T, El-Gilany AH, Elghannam D, Abdel-Ghaffar H, Saad MA, Sobh M: Amniotic fluid-derived mesenchymal stem cells cut short the acuteness of cisplatin-induced nephrotoxicity in Sprague-Dawley rats. Int $\mathbf{J}$ Stem Cells 2016;9:70-78.

19 Liu P, Feng Y, Dong D, Liu X, Chen Y, Wang Y, Zhou Y: Enhanced renoprotective effect of IGF-1 modified human umbilical cord-derived mesenchymal stem cells on gentamicin-induced acute kidney injury. Sci Rep 2016; 6:20287.

20 Rodrigues CE, Capcha JM, de Bragança AC, Sanches TR, Gouveia PQ, de Oliveira PA, Malheiros DM, Volpini RA, Santinho MA, Santana BA, Calado RD, Noronha IL, Andrade L: Human umbilical cord-derived mesenchymal stromal cells protect against premature renal senescence resulting from oxidative stress in rats with acute kidney injury. Stem Cell Res Ther 2017;8:19.

21 Zhang R, Yin L, Zhang B, Shi H, Sun Y, Ji C, Chen J, Wu P, Zhang L, Xu W, Qian H: Resveratrol improves human umbilical cord-derived mesenchymal stem cells repair for cisplatin-induced acute kidney injury. Cell Death Dis 2018;9:965.

22 Wu HJ, Yiu WH, Wong DWL, Li RX, Chan LYY, Leung JCK, Zhang Y, Lian Q, Lai KN, Tse HF, Tang SCW: Human induced pluripotent stem cell-derived mesenchymal stem cells prevent adriamycin nephropathy in mice. Oncotarget 2017;8:103640-103656.

$\checkmark 23$ Ko SF, Chen YT, Wallace CG, Chen KH, Sung PH, Cheng BC, Huang TH, Chen YL, Li YC, Chang HW, Lee MS, Yang CC, Yip HK: Inducible pluripotent stem cell-derived mesenchymal stem cell therapy effectively protected kidney from acute ischemia-reperfusion injury. Am J Transl Res 2018;10:3053-3067.
24 Pan XH, Zhou J, Yao X, Shu J, Liu JF, Yang JY, Pang RQ, Ruan GP: Transplantation of induced mesenchymal stem cells for treating chronic renal insufficiency. PLoS One 2017; 12:e0176273.

25 Monteiro BS, Santos BSD, Almeida BL, Hiura E, Fiorio WAB, Valdetaro GP, Gonçalves DV, Silva CS, Champion T, Campagnol D: Adipose tissue derived mesenchymal stem cell transplantation in the treatment of ische$\mathrm{mia}$ /reperfusion induced acute kidney injury in rats. Application route and therapeutic window. Acta Cir Bras 2018;33:1016-1026.

26 Ashour RH, Saad MA, Sobh MA, Al-Husseiny F, Abouelkheir M, Awad A, Elghannam D, Abdel-Ghaffar H, Sobh M: Comparative study of allogenic and xenogeneic mesenchymal stem cells on cisplatin-induced acute kidney injury in Sprague-Dawley rats. Stem Cell Res Ther 2016;7:126.

27 Yao W, Hu Q, Ma Y, Xiong W, Wu T, Cao J, Wu D: Human adipose-derived mesenchymal stem cells repair cisplatin-induced acute kidney injury through antiapoptotic pathways. Exp Ther Med 2015;10:468-476.

28 Elhusseini FM, Saad MA, Anber N, Elghannam D, Sobh MA, Alsayed A, El-Dusoky S, Sheashaa H, Abdel-Ghaffar H, Sobh M: Long term study of protective mechanisms of human adipose derived mesenchymal stem cells on cisplatin induced kidney injury in Sprague-Dawley rats. J Stem Cells Regen Med 2016;12:36-48.

29 Sheashaa H, Lotfy A, Elhusseini F, Aziz AA, Baiomy A, Awad S, Alsayed A, El-Gilany AH, Saad MA, Mahmoud K, Zahran F, Salem DA, Sarhan A, Ghaffar HA, Sobh M: Protective effect of adipose-derived mesenchymal stem cells against acute kidney injury induced by ischemia-reperfusion in Sprague-Dawley rats. Exp Ther Med 2016;11:1573-1580.

30 Zhang W, Liu L, Huo Y, Yang Y, Wang Y: Hypoxia-pretreated human MSCs attenuate acute kidney injury through enhanced angiogenic and antioxidative capacities. Biomed Res Int. 2014;2014:462472.

31 Xu Y, Shi T, Xu A, Zhang L: 3D spheroid culture enhances survival and therapeutic capacities of MSCs injected into ischemic kidney. J Cell Mol Med 2016;20:1203-1213.

32 Zhao J, Young YK, Fradette J, Eliopoulos N: Melatonin pretreatment of human adipose tissue-derived mesenchymal stromal cells enhances their prosurvival and protective effects on human kidney cells. Am J Physiol Renal Physiol 2015;308:F1474-1483.

-33 Sung PH, Chiang HJ, Wallace CG, Yang CC, Chen YT, Chen KH, Chen $\mathrm{CH}$, Shao PL, Chen YL, Chua S, Chai HT, Chen YL, Huang TH, Yip HK, Lee MS: Exendin-4-assisted adipose derived mesenchymal stem cell therapy protects renal function against co-existing acute kidney ischemia-reperfusion injury and severe sepsis syndrome in rat. Am J Transl Res 2017;9:3167-3183. 
34 Schubert R, Sann J, Frueh JT, Ullrich E, Geiger H, Baer PC: Tracking of adipose-derived mesenchymal stromal/stem cells in a model of cisplatin-induced acute kidney injury: comparison of bioluminescence imaging versus qRT-PCR. Int J Mol Sci 2018;19:2564.

>35 Peired AJ, Sisti A, Romagnani P: Mesenchymal stem cell-based therapy for kidney disease: a review of clinical evidence. Stem Cells Int 2016;2016:4798639.

>36 Moustafa FE, Sobh MA, Abouelkheir M, Khater Y, Mahmoud K, Saad MA, Sobh MA: Study of the effect of route of administration of mesenchymal stem cells on cisplatin-induced acute kidney injury in Sprague Dawley rats. Int J Stem Cells 2016;9:79-89.

\37 Asem M, Abdel-Megied AE-S, El-Mezayen HA, Nabil A, El-Karef A: Mesenchymal stem cells versus antioxidant therapy in acute renal failure induced by ischemia-reperfusion. Int J Recent Scientific Res 2017;8:18394-18402.

-38 Jang MJ, You D, Park JY, Kim K, Aum J, Lee C, Song G, Shin HC, Suh N, Kim YM, Kim CS: Hypoxic preconditioned mesenchymal stromal cell therapy in a rat model of renal ischemia-reperfusion injury: development of optimal protocol to potentiate therapeutic efficacy. Int J Stem Cells 2018;11:157-167.

>39 Liu X, Cai J, Jiao X, Yu X, Ding X: Therapeutic potential of mesenchymal stem cells in acute kidney injury is affected by administration timing. Acta Biochim Biophy Sin (Shanghai) 2017;49:338-348.

-40 Sherif IO, Al-Mutabagani LA, Alnakhli AM, Sobh MA, Mohammed HE: Renoprotective effects of angiotensin receptor blocker and stem cells in acute kidney injury: involvement of inflammatory and apoptotic markers. Exp Biol Med (Maywood) 2015;240:1572-1579.

-41 Havakhah S, Sankian M, Kazemzadeh GH, Sadri K, Bidkhori HR, Naderi-Meshkin H, Ebrahimzadeh Bideskan A, Niazmand S, Bahrami AR, Khajavi Rad A: In vivo effects of allogeneic mesenchymal stem cells in a rat model of acute ischemic kidney injury. Iran J Basic Med Sci 2018;21:824-831.

-42 Mohammadzadeh-Vardin M, Habibi Roudkenar M, Jahanian-Najafabadi A: Adenovirus-mediated over-expression of Nrf2 within mesenchymal stem cells (MSCs) protected rats against acute kidney injury. Adv Pharm Bull 2015;5:201-208.

43 Zhaleh F, Amiri F, Mohammadzadeh-Vardin M, Bahadori M, Harati MD, Roudkenar MH, Saki S: Nuclear factor erythroid-2 related factor 2 overexpressed mesenchymal stem cells transplantation, improves renal function, decreases injuries markers and increases repair markers in glycerol-induced acute kidney injury rats. Iran J Basic Med Sci 2016;19:323-329.
44 Geng X, Hong Q, Wang W, Zheng W, Li O, Cai G, Chen X, Wu D: Biological membrane-packed mesenchymal stem cells treat acute kidney disease by ameliorating mitochondrial-related apoptosis. Sci Rep 2017; 7:41136.

45 Si X, Liu X, Li J, Wu X: Transforming growth factor- $\beta 1$ promotes homing of bone marrow mesenchymal stem cells in renal ischemia-reperfusion injury. Int $\mathrm{J}$ Clin Exp Pathol 2015;8:12368-12378.

46 Liu N, Wang H, Han G, Cheng J, Hu W, Zhang J: Enhanced proliferation and differentiation of HO-1 gene-modified bone marrow-derived mesenchymal stem cells in the acute injured kidney. Int J Mol Med 2018; 42:946-956.

47 Burks SR, Nguyen BA, Tebebi PA, Kim SJ, Bresler MN, Ziadloo A, Street JM, Yuen PS, Star RA, Frank JA: Pulsed focused ultrasound pretreatment improves mesenchymal stromal cell efficacy in preventing and rescuing established acute kidney injury in mice. Stem Cells 2015;33:1241-1253.

-48 Lee KW, Kim TM, Kim KS, Lee S, Cho J, Park JB, Kwon GY, Kim SJ: Renal ischemia-reperfusion injury in a diabetic monkey model and therapeutic testing of human bone marrow-derived mesenchymal stem cells. J Diabetes Res 2018;2018:5182606.

49 Collino F, Pomatto M, Bruno S, Lindoso RS, Tapparo M, Sicheng W, Quesenberry P, Camussi G: Exosome and microvesicle-enriched fractions isolated from mesenchymal stem cells by gradient separation showed different molecular signatures and functions on renal tubular epithelial cells. Stem Cell Rev 2017;13:226-243.

50 Ragni E, Banfi F, Barilani M, Cherubini A, Parazzi V, Larghi P, Dolo V, Bollati V, Lazzari L: Extracellular vesicle-shuttled mRNA in mesenchymal stem cell communication. Stem Cells 2017;35:1093-1105.

-51 Grange C, Tapparo M, Bruno S, Chatterjee D, Quesenberry PJ, Tetta C, Camussi G: Biodistribution of mesenchymal stem cell-derived extracellular vesicles in a model of acute kidney injury monitored by optical imaging. Int J Mol Med 2014;33:1055-1063.

52 Ranghino A, Bruno S, Bussolati B, Moggio A, Dimuccio V, Tapparo M, Biancone L, Gontero P, Frea B, Camussi G: The effects of glomerular and tubular renal progenitors and derived extracellular vesicles on recovery from acute kidney injury. Stem Cell Res Ther 2017;8:24.

53 Yuan X, Li D, Chen X, Han C, Xu L, Huang T, Dong Z, Zhang M: Extracellular vesicles from human-induced pluripotent stem cell-derived mesenchymal stromal cells (hiPSC-MSCs) protect against renal ischemia/ reperfusion injury via delivering specificity protein (SP1) and transcriptional activating of sphingosine kinase 1 and inhibiting necroptosis. Cell Death Dis 2017;8:3200.
54 Ju GQ, Cheng J, Zhong L, Wu S, Zou XY, Zhang GY, Gu D, Miao S, Zhu YJ, Sun J, Du T: Microvesicles derived from human umbilical cord mesenchymal stem cells facilitate tubular epithelial cell dedifferentiation and growth via hepatocyte growth factor induction. PLoS One 2015;10:e0121534.

55 Zhang G, Zou X, Huang Y, Wang F, Miao S, Liu G, Chen M, Zhu Y: Mesenchymal stromal cell-derived extracellular vesicles protect against acute kidney injury through anti-oxidation by enhancing Nrf2/ARE activation in rats. Kidney Blood Press Res 2016;41:119_ 128.

\$56 Gu D, Zou X, Ju G, Zhang G, Bao E, Zhu Y: Mesenchymal stromal cells derived extracellular vesicles ameliorate acute renal ischemia reperfusion injury by inhibition of mitochondrial fission through miR-30. Stem Cells Int 2016;2016:2093940.

57 Zhu F, Chong Lee Shin OLS, Pei G, Hu Z, Yang J, Zhu H, Wang M, Mou J, Sun J, Wang Y, Yang Q, Zhao Z, Xu H, Gao H, Yao W, Luo X, Liao W, Xu G, Zeng R, Yao Y: Adipose-derived mesenchymal stem cells employed exosomes to attenuate AKI-CKD transition through tubular epithelial cell dependent Sox9 activation. Oncotarget 2017; 8:70707-70726.

58 Collino F, Bruno S, Incarnato D, Dettori D, Neri F, Provero P, Pomatto M, Oliviero S, Tetta C, Quesenberry PJ, Camussi G: AKI recovery induced by mesenchymal stromal cell-derived extracellular vesicles carrying microRNAs. J Am Soc Nephrol 2015; 26: 2349-2360.

59 Marcheque J, Bussolati B, Csete M, Perin L: Concise reviews: stem cells and kidney regeneration: an update. Stem Cells Transl Med 2019;8:82-92.

60 Zhao L, Hu C, Zhang P, Jiang H, Chen J: Novel preconditioning strategies for enhancing the migratory ability of mesenchymal stem cells in acute kidney injury. Stem Cell Res Ther 2018;9:225.

61 Ve eri -Haler, Cerar A, Perše M: (Mesenchymal) stem cell-based therapy in cisplatin-induced acute kidney injury animal model: risk of immunogenicity and tumorigenicity. Stem Cells Int 2017;2017:7304643.

62 Patschan D, Buschmann I, Ritter O, Kribben A: Cell-based therapies in acute kidney injury (AKI). Kidney Blood Press Res 2018; 43:673-681.

63 Fleig SV, Humphreys BD: Rationale of mesenchymal stem cell therapy in kidney injury. Nephron Clin Pract 2014;127:75-80.

64 Paliwal S, Chaudhuri R, Agrawal A, Mohanty $\mathrm{S}$ : Regenerative abilities of mesenchymal stem cells through mitochondrial transfer. J Biomed Sci 2018;25:31. 